\title{
Reservoir monitoring and characterization using satellite geodetic data: Interferometric Synthetic Aperture Radar observations from the Krechba field, Algeria
}

\author{
D.W.Vasco ${ }^{1}$, Alessandro Ferretti ${ }^{2}$, and Fabrizio Novali ${ }^{3}$ \\ ${ }^{1}$ Earth Sciences Division, Lawrence Berkeley National Laboratory, Berkeley, CA 94720 \\ ${ }^{2}$ Tele-Rilevamento Europa,T.R.E.s.r.l.,Via Vittoria Colonna,7-20149 Milano, Italy \\ ${ }^{3}$ Tele-Rilevamento Europa,T.R.E.s.r.l., Via Vittoria Colonna,7-20149 Milano, Italy
}




\title{
Reservoir monitoring and characterization using satellite geodetic data: Interferometric Synthetic Aperture Radar observations from the Krechba field, Algeria
}

\author{
D. W. Vasco *, Alessandro Ferretti ${ }^{\dagger}$, and Fabrizio Novali ${ }^{\dagger *}$
}

\begin{abstract}
Deformation in the material overlying an active reservoir is used to monitor pressure change at depth. A sequence of pressure field estimates, eleven in all, allow us to construct a measure of diffusive travel time throughout the reservoir. The dense distribution of travel time values means that we can construct an exactly linear inverse problem for reservoir flow properties. Application to Interferometric Synthetic Aperture Radar (In$\mathrm{SAR}$ ) data gathered over a $\mathrm{CO}_{2}$ injection in Algeria reveals pressure propagation along two northwest trending corridors. An inversion of the travel times indicates the existence of two northwest-trending high permeability zones. The high permeability features trend in the same direction as the regional fault and fracture zones. Model parameter resolution estimates indicate that the features are well resolved.
\end{abstract}

\section{INTRODUCTION}

It has long been recognized that reservoir and aquifer production can lead to deformation of the overburden (Castle et al. 1969, Colazas 1971, Geertsma 1973, Evans et al. 1982, Segall 1985, Palmer 1990, Dussealt et al. 1993, Chilingarian et al. 1994, Bruno and Bilak 1994, Castillo et al. 1997, Massonnet et al. 1997, Vasco et al. 1998, Mossop and Segall 1999, Stancliffe and van der Kooij 2001). These studies utilized geodetic data, such as leveling measurements, in order to quantify surface deformation related to fluid production or injection. Currently, time-lapse seismic surveys are providing additional deformation measurements, distributed throughout the overburden (Guilbot and Smith 2002, Landro and Stammeijer 2004, Tura et al. 2005, Hatchell and Bourne 2005, Barkved and Kristiansen 2005, Hall 2006, Roste et al. 2006, Rickett et al.
2007, Staples et al. 2007). Recently, the observed deformation has been used to better understand fluid flow and the heterogeneity of flow properties within the reservoir (Vasco et al. 2000a, Vasco et al. 2001, Du et al. 2005, Vasco and Ferretti 2005, Hodgson et al. 2007, Vasco et al. 2008).

In this paper we apply the method described in Vasco et al. (2008) to a set of Interferometric Synthetic Aperture Radar (InSAR) range change data associated with the injection of $\mathrm{CO}_{2}$. The approach is based upon the arrival time of a propagating diffusive disturbance (Virieux et al. 1994, Vasco et al. 2000b, Shapiro et al. 2002). As in Vasco et al. (2008) the pressure propagation is constrained by InSAR observations gathered by an orbiting satellite (Burgmann et al. 2000). The advantages of this technique are that it uses remotely gathered data, keeping expenses low, it is relatively insensitive to the heterogeneity of mechanical properties within the reservoir, and it leads to a linear inverse problem for flow properties. We should note that the method can be used on pressure changes estimated directly from time-lapse seismic observations, as discussed in Landro (2001) and Hoversten et al (2003).

\section{METHODOLOGY}

If a sufficient volume of fluid is withdrawn from or injected into a reservoir, the reservoir can deform, for example undergoing volume change. The deformation within the reservoir induces displacements within the surrounding medium, in some cases producing measurable deformation at the Earth's surface. Our goal is to infer flow and flow properties, such as permeability, from measurements of overburden deformation. The approach described here involves three main steps. First, the time-varying deformation is used to estimate volume and pressure changes within the reservoir as a function of both space and time 
(Vasco et al. 2001, Vasco et al. 2008). Second, the time-varying volume and/or pressure changes determine 'arrival times' associated with a propagating fluid disturbance within the reservoir (Vasco et al. 2000b, Vasco 2004, Vasco et al. 2008). Finally, flow properties, in particular the effective permeabilities, are derived from the pressure 'arrival times'.

\section{Estimating Volume and Pressure Changes within the Reservoir}

In the first step we utilize estimates of overburden deformation, as produced by geodetic techniques (Evans et al. 1982, Vasco e al. 1988, Dussealt et al. 1993, Bruno and Bilak 1994, Castillo et al. 1997, Massonnet et al. 1997, Fielding et al. 1998, Mossop and Segall 1999, Vasco et al. 2000a, Stancliffe and van der Kooij 2001, Schmidt and Burgmann 2003, Vasco and Ferretti 2005) or by timelapse seismic monitoring (Guilbot and Smith 2002, Landro and Stammeijer 2004, Tura et al. 2005, Hall 2006, Roste et al. 2006, Rickett et al. 2007, Staples et al. 2007) to constrain volume and fluid pressure changes within a reservoir. Our analysis assumes that a set of deformation measurements have been gathered. The observations might consist of leveling data (Vasco et al. 2000a), tilts (Castillo et al. 1997, Wright 1998, Wright et al. 1998, Du et al. 2005), InSAR range change observations (Massonnet and Feigl 1998, Burgmann et al. 2000, Stancliffe and van der Kooij 2001), Global Positioning Satellite measurements (GPS) (Spiess et al. 1998), time-lapse seismic time strains (Hatchell and Bourne 2005, Rickett et al. 2007), or accurate seafloor pressure observations (Sasagawa et al. 2003).

\section{Estimation of volume change}

In order to map the deformation measurements, denoted by $\mathbf{u}(t)$, as well as any possible fluid pressure data $\delta \mathbf{p}(t)$ into volume change within the reservoir, $\mathbf{v}_{f}(t)$ we adopt the coupled inversion method described in Vasco et al. (2001). The components of the vector $\mathbf{v}_{f}(t)$ represent the fractional volume change of each grid block of a reservoir model. Outside the reservoir, the Earth is assumed to deform elastically over the time interval of interest. Thus, the relationship between the overburden deformation and reservoir fluid pressure changes and the reservoir volume changes may be written as a linear system of equations

$$
\left[\begin{array}{c}
\mathbf{u}(t) \\
\delta \mathbf{p}(t)
\end{array}\right]=\left[\begin{array}{c}
\mathbf{\Upsilon} \\
\boldsymbol{\Pi}
\end{array}\right] \mathbf{v}_{f}(t)
$$

where the coefficients of the matrices $\boldsymbol{\Upsilon}$ and $\boldsymbol{\Pi}$ are given by integrals of the Green's function $g_{i}\left(\mathbf{x}_{k}, \mathbf{x}^{\prime}\right)$

$$
\Upsilon_{i j k}=\int_{V_{j}} g_{i}\left(\mathbf{x}_{k}, \mathbf{x}^{\prime}\right) d V
$$

for observation point $\mathbf{x}_{k}$, integration variable $\mathbf{x}^{\prime}$, deformation components $i$, and reservoir grid block $j$ with volume
$V_{j}$, and

$$
\Pi_{j k}=C_{e}^{-1}\left[\delta_{j k}-\frac{B}{\rho} \int_{V_{j}} G_{i i}\left(\mathbf{x}_{k}, \mathbf{x}^{\prime}\right) d V\right]
$$

respectively, where $B$ is Skempton's pore pressure coefficient (Rice and Cleary 1976, Wang and Kuempel 2003) and $\rho$ is the density. The integrand in $(2), g_{i}\left(\mathbf{x}_{k}, \mathbf{x}^{\prime}\right)$, is the Green's function which is the point response of the elastic medium at location $\mathbf{x}_{k}$. As such, it is medium-dependent, as discussed in Vasco et al. (2000a). The integral is evaluated over the volume of the $j$-th grid block, $V_{j}$. The kernel $G_{i i}$ in the integral in (3) is constructed from the Green's function solution $g_{i}$, as described in Segall (1985)

$$
G_{i i}=(2 \mu+\lambda) \frac{\partial g_{i}}{\partial x_{i}}
$$

where $\mu$ is the shear modulus, and $\lambda$ is a Lame parameter, and we follow the summation convention, summing over the repeated index variable $i$. For a homogeneous elastic half-space the Green's function takes a particularly simple form (Segall 1985, Vasco et al. 1988).

The system of equations (1) comprises the linear inverse problem for volume change within the reservoir, $\mathbf{v}_{f}$. For simple reservoirs, such as a single layer of grid blocks, the inverse problem can be well-determined. For more complicated structures the inverse problem may be under-determined. As described in Vasco et al. (2001) and Vasco and Ferretti (2005), we regularize the inverse problem by invoking norm and roughness penalties. In addition, we can also penalize volume change as a function of distance from the injection well on the assumption that the largest pressure and volume change should be near the well (Vasco et al. 2001). We can also constrain the total volume change to be similar to the total injected or withdrawn fluid volume (Vasco and Ferretti 2005).

Mapping estimates of volume change into changes in pressure and overburden stress

If the reservoir behaves elastically, the estimates of reservoir volume change can be mapped directly into pressure change within the reservoir. The relationship, given by Segall (1985), includes two terms,

$$
\delta p(\mathbf{x}, t)=C_{\nu} v_{f}(\mathbf{x}, t)-\frac{B^{2}}{3 \rho} \int_{V} G_{i i}\left(\mathbf{x}, \mathbf{x}^{\prime}\right) v_{f}\left(\mathbf{x}^{\prime}, t\right) d V
$$

where

$$
C_{\nu}=\frac{B^{2}}{\rho}\left[\mu \frac{2}{3} \frac{\left(1+\nu_{d}\right)\left(1+\nu_{u}\right)}{\left(\nu_{u}-\nu_{d}\right)}-3 K_{u}\right],
$$

$\nu_{u}$ and $\nu_{d}$ are the undrained and drained Poisson's ratios (Rice and Cleary 1976), $K_{u}$ is the undrained bulk modulus. The first term is the direct elastic mapping from volume change to pressure change. The second term in equation (5) accounts for propagation of stress within the overburden. That is, stress changes in the overburden will stress the reservoir, resulting in deformation some distance from regions of volume change (Segall 1985). 


\section{A Diffusion Equation for Fluid Pressure Variations within the Reservoir}

Having determined the pressure changes within the reservoir we now wish to infer flow properties such as permeability. The total fluid pressure, $p(\mathbf{x}, t)$ is obtained by adding the pressure changes to the background pressure $p_{0}(\mathbf{x})$ which we assume is changing so slowly that its time dependence can be neglected relative to the time dependence of $\delta p$,

$$
p(\mathbf{x}, t)=p_{0}(\mathbf{x})+\delta p(\mathbf{x}, t)
$$

While it is possible to work directly with the pressure estimates themselves to image permeability (Vasco et al. 2001), we will adopt a different approach, based upon the idea of a diffusive arrival time (Virieux et al. 1994, Vasco et al. 2000b, Shapiro et al. 2002, Vasco et al. 2008). One advantage of this approach is that, for an elastic medium, the arrival time is less sensitive to the variations of mechanical properties of the medium. That is, in the absence of visco-elastic or memory effects, the arrival time, a kinematic quantity, will not depend on the strength of the coupling between pressure and volume change. Another advantage is that, as shown below, the inverse problem becomes linear if the arrival times can be estimated over the region of interest.

Our starting point is the set of partial differential equations describing the flow of an aqueous phase and a nonaqueous phase (Peaceman, 1977)

$$
\begin{gathered}
\nabla \cdot\left[\frac{\rho_{w} K(\mathbf{x}) k_{r w}}{\mu_{w}} \nabla\left(p_{w}(\mathbf{x}, t)-\rho_{w} g z\right)\right]=\frac{\partial\left(\phi \rho_{w} S_{w}\right)}{\partial t} \\
\nabla \cdot\left[\frac{\rho_{n} K(\mathbf{x}) k_{r n}}{\mu_{n}} \nabla\left(p_{n}(\mathbf{x}, t)-\rho_{n} g z\right)\right]=\frac{\partial\left(\phi \rho_{n} S_{n}\right)}{\partial t}+q
\end{gathered}
$$

where $S_{w}$ and $S_{n}$ denote the saturations of the aqueous and non-aqueous phases respectively and $q$ denotes the injection rate of the non-aqueous phase. The relative permeabilities of the aqueous and non-aqueous phases are represented by $k_{r w}$ and $k_{r n}$ while the absolute permeability is given by $K(\mathbf{x})$. The respective densities are $\rho_{w}$ and $\rho_{n}$, the gravitational constant is $g$ and the porosity is $\phi(\mathbf{x})$. The pressure associated with the aqueous phase is $p_{w}(\mathbf{x}, t)$ while the pressure for the non-aqueous phase is $p_{n}(\mathbf{x}, t)$, the viscosities are $\mu_{w}$ and $\mu_{n}$. The above equations are coupled because the saturations are assumed to sum to unity

$$
S_{w}+S_{n}=1 .
$$

As noted in Vasco et al. (2008), one can derive an equation for the total pressure fluid, $p$, from the above equations:

$$
C_{e} \frac{\partial p}{\partial t}-\nabla \cdot[K \nabla p]+\hat{q}=0
$$

assuming that the transient pressure change propagates significantly faster than the $\mathrm{CO}_{2}$ saturation front. Applying the Fourier transform to equation (8), one has the frequency domain equivalent,

$$
\nabla^{2} P+\boldsymbol{\Lambda} \cdot \nabla P-i \omega \kappa P=\hat{Q}
$$

where $\boldsymbol{\Lambda}$ is the gradient of the logarithm of conductivity,

$$
\mathbf{\Lambda}(\mathbf{x})=\nabla \ln K(\mathbf{x}),
$$

which vanishes for constant $K(\mathbf{x})$, and

$$
\kappa(\mathbf{x})=\frac{C_{e}(\mathbf{x})}{K(\mathbf{x})}
$$

is the reciprocal of the diffusivity, the quantity $\hat{Q}$ is the Fourier transform of the source term.

\section{Asymptotic Solution of the Diffusion Equation}

As in hyperbolic wave propagation (Kline and Kay 1979, Aki and Richards 1980, Kravtsov and Orlov 1990), an expression for the 'arrival time' of a pressure disturbance follows from an asymptotic or 'high-frequency' solution of the diffusion equation (9) (Cohen and Lewis 1967, Virieux et. al. 1994, Vasco et al. 2000b). An asymptotic solution to the diffusion equation (9) is a power series in $1 / \sqrt{\omega}$,

$$
P(\mathbf{x}, \omega)=e^{-\sqrt{-i \omega} \sigma(\mathbf{x})} \sum_{n=0}^{\infty} \frac{A_{n}(\mathbf{x})}{(\sqrt{-i \omega})^{n}}
$$

a specific case of the general form of Friedlander and Keller (1955). The form of the series (12) may be deduced on physical grounds, by considering a large argument expansion of the modified Bessel function of zeroth order, the solution to the diffusion equation for a homogeneous medium, (Virieux et al. 1994). For the most rapidlyvarying component of pressure, for large values of $\omega$, the zeroth-order term

$$
P(\mathbf{x}, \omega)=A_{0}(\mathbf{x}) e^{-\sqrt{-i \omega} \sigma(\mathbf{x})},
$$

or its time-domain equivalent, obtained by inverse Fourier transforming equation (13),

$$
p(\mathbf{x}, t)=A_{0}(\mathbf{x}) \frac{\sigma(\mathbf{x})}{2 \sqrt{\pi t^{3}}} e^{-\sigma^{2}(\mathbf{x}) / 4 t},
$$

(Virieux et al. 1994, Vasco et al. 2000b), will accurately represent the solution to the diffusion equation. The expression for $p(\mathbf{x}, t)$ depends on two functions, $\sigma(\mathbf{x})$, which is the phase or pseudo-phase function, and on $A_{0}(\mathbf{x})$, the amplitude function. As shown in Virieux et al. (1994) and Vasco et al. (2000b) these functions are obtained by substituting the asymptotic expression for pressure into the governing equation (9).

The Eikonal Equation, Trajectories, and the Arrival Time of the Maximum Pressure Change

The equation governing the evolution of the phase, $\sigma(\mathbf{x})$, is obtained by substituting the power series (12) into the diffusion equation (9). For the high frequency component of the pressure disturbance, the terms of highest order in $\omega,\left[(\sqrt{-i \omega})^{2} \sim \omega\right]$ will dominate and we obtain

$$
\nabla \sigma(\mathbf{x}) \cdot \nabla \sigma(\mathbf{x})-\kappa(\mathbf{x})=0
$$


(Cohen and Lewis 1967, Virieux et al. 1994, Vasco et al. 2000b). Equation (15), known as the eikonal equation, appears in studies of high-frequency wave propagation (Kline and Kay 1979, Aki and Richards 1980). The non-linear partial differential equation (15) is equivalent to a system of two ordinary differential equations, the characteristic equations (Courant and Hilbert 1962). In the method of characteristics, solutions are developed along particular trajectories, which are denoted by $\mathbf{X}(l)$, where $l$ signifies position along the curve. The equations for the characteristic curves are a set of ordinary differential equations which depend upon $\sqrt{\kappa}$, the ray equations,

$$
\begin{aligned}
& \frac{d \mathbf{X}}{d l}=\nabla \sigma \\
& \frac{d \sigma}{d l}=\sqrt{\kappa}
\end{aligned}
$$

(Courant and Hilbert 1962). From equation (17), we can write the phase function as an integral

$$
\sigma(\mathbf{x})=-\int_{\mathbf{X}(l)} \sqrt{\kappa} d l
$$

where $\mathbf{X}(l))$ is the trajectory from the injection well to the observation point $\mathbf{x}$.

As pointed out by Virieux et al. (1994) a physical interpretation of the phase function, $\sigma(\mathbf{x})$, follows from the zeroth-order expression (14). In particular, if we differentiate the expression for $p(\mathbf{x}, t)$ with respect to $t$ and set the derivative equation to zero, we find that

$$
\sigma(\mathbf{x})=\sqrt{6 T_{\max }(\mathbf{x})}
$$

where $T_{\max }(\mathbf{x})$ is the time as which the pressure derivative is a maximum at the point $\mathbf{x}$. Thus, we can estimate $\sigma(\mathbf{x})$ directly from a sequence of pressure estimates or from the result of a reservoir simulation. For example, we can use estimates of $p(\mathbf{x}, t)$ obtained from the inversion of deformation data to compute $\sigma(\mathbf{x})$ (Vasco et al. 2008). From the time series of pressure estimates for each grid block of the reservoir model, we can estimate the rate of change of the pressure and estimate $T_{\max }(\mathbf{x})$ and hence $\sigma(\mathbf{x})$. Alternatively, we can deconvolve the source-time function using injection rate data and calculate $T_{\max }(\mathbf{x})$ from the peak of the transient pressure variation in each grid block.

\section{A Linear Inversion Algorithm for Flow Prop- erties within the Reservoir}

As noted above, deformation of the overburden can be mapped into reservoir volume or fluid pressure changes, $\delta p(\mathbf{x}, t)$, for a sequence of time intervals. Given sufficient temporal sampling, we can compute pressure arrival times from the sequence of estimates $\delta p(\mathbf{x}, t)$. We denote the arrival times at the $i$-th grid block by $\Sigma_{i}$. The characteristic equations and the resulting integral expression for the phase (18) provides a relationship between the estimated phase $\Sigma_{i}$ and the flow properties contained in $\kappa$,

$$
\Sigma_{i}=-\int_{\mathbf{X}_{i}} \sqrt{\kappa} d l
$$

where $\mathbf{X}_{i}$ denotes the trajectory associated with the $\Sigma_{i}$ observation point. The trajectory is computed using the sampled version of equation (16)

$$
\frac{d \mathbf{X}}{d l}=\nabla \Sigma
$$

where $\Sigma$ is obtained from the reservoir pressure estimates. Thus, the trajectory is known a priori, computed directly from the observations. The fact that the trajectory is specified and is not an unknown in the problem linearizes the inverse problem for $\sqrt{\kappa}$. Thus, given a collection of arrival times, the collection of equations of the form (20) produces a linear system

$$
\Sigma=\mathbf{G y}
$$

which may be solved for $\mathbf{y}$, where $y_{i}=\sqrt{\kappa_{i}}$. To further understand how knowledge of $\Sigma(\mathbf{x})$ in the region of interest linearizes the inverse problem, consider the sampled form of the eikonal equation

$$
\nabla \Sigma(\mathbf{x}) \cdot \nabla \Sigma(\mathbf{x})=\kappa(\mathbf{x})
$$

If we have estimates for $\Sigma(\mathbf{x})$ in the reservoir region then (23) provides a direct linear expression for $\kappa(\mathbf{x})$ in terms of $|\nabla \Sigma(\mathbf{x})|^{2}$.

Because of errors in the data, modeling approximations, as well as other difficulties, such as non-uniqueness, it is not advisable to solve the linear system (22) directly. Rather, a penalized, least-squares approach (Lawson and Hanson 1974) leads to a more robust solution (Parker 1994). Thus, we minimize a linear combination of the sum of the squares of the residuals and penalty terms related to the norm of deviations from a prior model $\mathbf{y}_{0}$ and a roughness penalty

$$
R=|\boldsymbol{\Sigma}-\mathbf{G} \mathbf{y}|_{2}+W_{n}\left|\mathbf{y}-\mathbf{y}_{0}\right|_{2}+W_{r}|\mathbf{D y}|_{2}
$$

where $|\mathbf{z}|_{2}$ signifies the $L_{2}$ norm of the vector $\mathbf{z}, W_{n}$ and $W_{r}$ are scalar coefficients controlling the importance of the penalty terms in relation to the importance of fitting the data, and $\mathbf{D}$ is a matrix which approximates a differencing operator. All the terms in the sum (24) are quadratic and hence the necessary conditions for a minimum of $R$ is a linear system of equations which is solved using an efficient iterative algorithm (Paige and Saunders 1982).

Because the inverse problem for flow properties, as contained in the vector $\mathbf{y}$, is linear we have well established techniques for model assessment. For example, we can compute the model parameter resolution matrix (Aki and Richards 1980, Parker 1994) in order to explore the spatial resolution of our estimates. The model parameter resolution matrix relates our model parameter estimates, $\hat{\mathbf{y}}$, obtained by minimizing the penalized misfit functional (24), to a hypothetical 'true' model which satisfied the constraint equations (22). Because the necessary equations for a minimum of $R$ are linear, we can write the solution as

$$
\hat{\mathbf{y}}=\mathbf{G}^{\dagger} \boldsymbol{\Sigma}
$$


where $\mathbf{G}^{\dagger}$ is the 'generalized inverse' of $\mathbf{G}$ (Aki and Richards 1980). The resolution matrix, $\mathbf{R}$, is given by

$$
\mathbf{R}=\mathbf{G}^{\dagger} \mathbf{G}
$$

and relates $\hat{\mathbf{y}}$ to $\mathbf{y}$

$$
\hat{\mathbf{y}}=\mathbf{R y} .
$$

It is obtained by substituting the expression for $\boldsymbol{\Sigma}$, equation (22), into equation (25). The rows of the resolution matrix are averaging coefficients, indicating the contribution of all model parameters on the model parameter estimate. For a perfectly resolved model the resolution matrix would be an identity matrix.

\section{APPLICATION: $\mathrm{CO}_{2}$ INJECTION AT THE KRECHBA FIELD, ALGERIA}

\section{Geology and Field History}

The Krechba field in Algeria is defined by the structural high of a northwest trending anticline. Gas produced from this field and two nearby fields contains $\mathrm{CO}_{2}$ concentrations ranging from 1 to $9 \%$ which is above the export gas specification of $0.3 \%$. The $\mathrm{CO}_{2}$ from the three fields is separated from the hydrocarbons and reinjected into three adjacent wells, KB-501, KB-502, and KB-503 at rates of the order of tens of millions of cubic feet per day. The injection is restricted to a 20 meter thick layer, some 2,000 meters deep. The reservoir is overlain by over 1,000 meters of shale which forms a significant barrier to flow. Berkeley Laboratory and Tele-Rilevamento (TRE), in partnership with British Petroleum, examined the utility of satellite range change data for monitoring the reservoir during $\mathrm{CO}_{2}$ injection. Of particular interest were the identification of features controlling flow and the possibility of detecting $\mathrm{CO}_{2}$ migration out of the reservoir and into the surrounding formation.

Because the reservoir is initially water-filled, the injection of $\mathrm{CO}_{2}$ into the water column induces multiphase flow. The $\mathrm{CO}_{2}$ behaves super-critically at reservoir pressures, with a viscosity and density only moderately different from that of water. The injection pressure was roughly 120 Bars, resulting in a density greater than 0.85 grams $/ \mathrm{cm}^{3}$. In the study region the reservoir depth does not vary significantly, and the density differences do not impact the flow. We model the $\mathrm{CO}_{2}$-water system as an equivalent single phase for the pressure calculations. This approximation should be sufficient given the reservoir pressure conditions and the time interval (Kumar et al. 2005).

\section{Data Analysis and Range Change Estimates}

We utilized satellite radar images of the European Space Agency (ESA) Envisat archive from July 12, 2003 through March 19, 2007 to monitor surface deformation during the injection. The satellites reflect radar waves off the surface of the Earth. Upon successive passes, the phase change between the backscattered radar energy is used to measure minute changes in distance between the satellite and the Earth's surface (Massonnet and Feigl 1998, Burgmann et al. 2000). Two satellite tracks, number 65 and number 294, traversed the region during the $\mathrm{CO}_{2}$ injection, containing 26 and 18 images, respectively. The data were processed using a permanent scatterer technique developed by Politenico di Milano and TRE (Ferretti et al. 2000, 2001). In this approach, stable scatterers are identified using a statistically-based analysis of the phase and amplitude characteristics of the energy scattered from the Earth's surface. A subset of scatterers are identified as Permanent Scatterer Candidates and used to estimate atmospheric and orbital errors. The atmospheric and orbital biases in the signal phase are corrected, and estimates of range change are derived from the phase shifts between pairs of back-scattered radar signals. The range change represents the change in distance from a point on the Earth's surface and the nominal satellite orbit.

In this study we focus on range change associated with $\mathrm{CO}_{2}$ injection at well KB-502. To date, this well has accounted for $38 \%$ of injected $\mathrm{CO}_{2}$ even though injection started in April 2005, eight months after activity in KB501 and KB-503. There is a clear range change signal in both tracks, as indicated in Figure 1. There is considerable scatter in the range velocity estimates, and evidence of an artifact, due to the presence of a stream bed cutting across the anomaly. Because injector KB-502 did not begin operation until April 2005 and the data from both tracks 65 and 294 were available until December 2006, we had roughly 20 months of InSAR monitoring data.

In order to improve the signal-to-noise ratio, we stacked the data from the two tracks and averaged the values in both space and time. Track 65 contained 24 usable images extending from July 2003 to December 2006 while Track 294 contained 17 usable images from December 2003 until March 2007. The two sets of images were interpolated in time onto 11 images from the time of injection until 545 days later $[0,24,58,96,128,159,198,265,306,408,545$ days]. The spatial grid of values was averaged in a 10 element moving window and re-interpolated onto a 150 by 150 grid of pixels. The resulting range velocity estimates, shown in Figure 2, represent the average range change per year for the time interval July 2003-March 2007. From this figure it appears that there are two northwest trending lobes of range decrease, possibly representing uplift due to reservoir pressure changes. The peak range change overlies the injection well trace, which is indicated by the solid line in Figure 2. Because of the averaging, the peak velocity in Figure 2 is less than the peak values of each component, shown in Figure 1. Even though we made every effort to remove permanent scatterers associated with the stream bed, a hint of the artifact is still evident in the average range velocity estimates. Actual range changes for various time intervals are shown in Figure 3. After 24 days of injection a trend of range decrease underlies the horizontal injection well. In addition, a lobe of northwest 
trending range decrease extends from the eastern edge of the injector. The two lobed pattern is evident after 96 days of injection and seems to migrate to the northwest.

\section{Estimation of Reservoir Pressure Changes}

The first step in the inversion algorithm involves estimating reservoir volume change, based upon the surface deformation data, in our case the satellite range change data. Expression (1) is the operative equation with the pressure terms set equal to zero because we have no reservoir pressure data

$$
\mathbf{u}(t)=\Upsilon_{\mathbf{v}_{f}}(t)
$$

The reservoir was modeled as a single layer of grid blocks, lying at a depth of $2 \mathrm{~km}$. Initially, the layer was assumed to be twenty meters thick, the average thickness of the reservoir. The layer was sub-divided into a grid of 50 by 50 cells and the fractional volume change associated each cell was an unknown. Both roughness and model norm penalty terms were included in the inversion. Furthermore, we included a term which penalized volume change as a function of distance from the injection well (Vasco et al. 2001). Volume change was mapped into pressure based upon equation (5). The effect of stress propagation through the overburden, the second term in equation (5) was neglected because numerical tests indicated that it was of second order in importance. Hence, we used the relationship

$$
\delta p(\mathbf{x}, t)=C_{\nu} v_{f}(\mathbf{x}, t)
$$

to map the volume change into the corresponding pressure change.

As an example, in Figure 4 we display pressure change within the reservoir after 545 days of injection. Note that the pressure variation within the reservoir only contains a single northwest trending lobe of pressure increase. This does not agree with the actual range change values after 545 days of injection (Figure 3). Various combinations of weighting parameters were tried in an attempt to match the pattern or range change. After numerous tries, we were unable to obtain a continuous pressure variation containing two northwest trending lobes if we required the volume and pressure changes to lie within the reservoir interval. If the zone of volume change extended up to 100 or 200 meters from the center of the layer we could produce a smooth model with two lobes of volume change (Figure 5). Our final model allowed for volume change up to 190 meters from the center of the reservoir layer. This extension of volume change above the reservoir may not be absolutely necessary to fit the observations. In particular, we have not run tests to see if it is necessary to extend the volume change beyond the reservoir boundaries in order to fit the geodetic observations. Given that the threedimensional seismic data does indicate that near vertical faults do cut the reservoir interval, it is certainly possible for injected fluid to migrate out of the reservoir. In order to determine it such migration is indeed occurring, it is necessary to incorporate additional data, such as microseismicity. Four snapshots of pressure change are shown in Figure 5. Note the decrease in pressure change near the middle of each lobe. This is most likely an artifact, due to the influence of the stream bed which reduced the range change in this region (Figure 3). In conclusion, there is the suggestion that the injected fluids may be migrating into northwest trending fault and fracture zones.

\section{Estimation of Reservoir Permeability Vari- ations}

The final step involves estimating the diffusive travel time associated with the pressure disturbance and to invert for flow properties. The pressure change from the background values in each grid block were interpolated using low-order polynomials. The time at which the pressure change was a maximum was found for each grid block of the model. The resulting phase function is shown in Figure 6 and the sampled phase values are shown in Figure 7. Because of the artifacts associated with the stream beds noted above, the arrival time field in Figure 6 contains local maxima on each lobe. An additional lateral smoothing of the phase field removed these artifacts and enable us to compute the trajectories in a robust fashion. The trajectories, shown in Figure 7, were computed using equation (21), that is, by simply marching down the gradient from the observation point to the injection well. Because the phase estimates are available over a region surrounding the injection well, we can calculate the gradient $\nabla \Sigma$ and estimate the trajectories directly. Thus, we can fix the path $\mathbf{X}_{i}$ in the integral (20) resulting in a linear relationship between $\Sigma_{i}$ and $\sqrt{\kappa}$. Corresponding to the phase estimates shown in Figure 7 there is an associated linear system of equations (22). Formulating the linear system as a penalized least squares problem, see equation (24), we form the necessary equations for a minimum. We can solve this associated linear system using an iterative solver which takes advantage of the sparsity of the system (Paige and Saunders 1982). The resulting solution, shown in Figure 8, contains two high permeability zones extending to the north and west of the injection well. The two features coincide with the lobes seen in the range change observations. The zones are sub-parallel to the axis of the anticline and sub-parallel to faults and fractures which are observed in the area. The fit to the phase data is shown in Figure 9. Generally, the predicted values are close to the observed values, lying near the $45^{\circ}$ line which signifies an exact match.

\section{Model Parameter Resolution}

The fact that the inverse problem is linear means that we are free to use techniques from geophysical inverse theory (Parker 1994). In particular, we can compute the model parameter resolution matrix $\mathbf{R}$, as formulated in equation (26). As noted previously, for a perfectly resolved model the matrix $\mathbf{R}$ would be the identity matrix. The diagonal elements of the resolution matrix are a convenient 
measure of ones ability to recover model parameter values in isolation from the others. Specifically, a diagonal value near 1 means that it is possible to estimate the parameter with little or no tradeoff from the other model parameters. Conversely, a value near 0 means that there is significant trade-off with other parameters. The resolution corresponding to our inverse problem is shown in Figure 10. Where we have phase estimates, as indicated in Figure 7 , the resolution is rather good, with values near 1. In the areas in which there are no phase values, the resolution is zero or nearly zero. The resolution estimates shown in this figure only correspond to the resolving power associated with the arrival time values. In particular, the diagonal components of the resolution matrix do not account for the smoothing and averaging that was used to estimate the range change values and smoothing due to the mapping of the deformation estimates into pressure arrival time estimates.

\section{CONCLUSIONS}

Time-lapse geodetic data provide a means to image flow and to infer flow properties such as permeability. It is advantageous to have sufficient temporal as well as spatial sampling. The time sampling allows us to implement an arrival time inversion method which is less sensitive to the strength of the coupling between volumetric deformation and pressure change. Dense spatial sampling means that we can fix the trajectories used in the arrival time inversion, exactly linearizing the problem. The technique described here is applicable to a wide variety of data types including InSAR, GPS, tilt, precision ocean bottom pressure sensors, and time-strains from time-lapse seismic surveys. Furthermore, the method can be used on pressure changes estimated directly from time-lapse seismic observations.

The approach appears to work well in practice, as illustrated by our application to InSAR data related to a $\mathrm{CO}_{2}$ injection at the Krechba field in Algeria. It appears that the pressure change due to the start of injection propagated to the northwest along two high-permeability pathways. The orientation of the high-permeability features agrees with the trend of faults and fractures in the region. Furthermore, the permeability estimates are well resolved, as indicated by the model parameter resolution computations. In order to fit the InSAR range change data with a smoothly varying model containing two northwest trending lobes of pressure, change extended some 100 to 200 meters above the reservoir layer. This may be due to pressure change outside the reservoir interval or perhaps due to an element of the modeling, such as the significant lateral extent of the grid blocks. Additional observations, such as micro-seismic surveys, are required to resolve the depth extent of the pressure change.

\section{ACKNOWLEDGMENTS}

This work was supported by the Assistant Secretary, Office of Basic Energy Sciences and the GEOSEQ project for the Assistant Secretary for Fossil Energy, Office of Coal and Power Systems through the National Energy Technology Laboratory of the U. S. Department of Energy under contract DE-AC02-05CH11231. The In Salah $\mathrm{CO}_{2}$ Joint Industry Project (BP, StatoilHydro, and Sonatrach) is thanked for the provision of production, injection, and subsurface data. The permanent scatterer (PS) data were processed by Tele-Rilevamento Europa (TRE), a spin-off company of Politecnico di Milano, world-wide exclusive licensee of the Polimi PS Technique ${ }^{\mathrm{TM}}$. The authors wish to thank ESA for all satellite data used in this study and the entire TRE staff for supporting the SAR data processing. Additional computations were carried out at the Center for Computational Seismology, Berkeley Laboratory. 


\section{REFERENCES}

Aki, K., and P. G. Richards, 1980, Quantitative seismology: Freeman and Sons.

Barkved, O. I., and T. Kristiansen, 2005, Seismic timelapse effects and stress changes: Examples from a compacting reservoir: The Leading Edge, 24, 1244-1248.

Bruno, M. S., and R. A. Bilak, 1994, Cost-effective monitoring of injected steam migration using surface deformation analysis: SPE Western Regional Meeting., 27888, 397-412.

Burgmann, R., R. A. Rosen, and E. J. Fielding, 2000, Synthetic aperture radar interferometry to measure Earth's surface topography and its deformation: Annual Reviews of Earth and Planetary Sciences, 28, 169-209.

Castillo, W., S. Hunter, P. Harben, C. Wright, R. Conant, and E. Davis, 1997, Deep hydraulic fracture imaging: Recent advances in tiltmeter technologies: International Journal of Rock Mechanics and Mining Science, 34, 3-4.

Castle, R. O., R. F. Yerkes, and F. S. Riley, 1969, A linear relationship between liquid production and oil-related subsidence: in Tison, L. J., (Ed.), Land subsidence, 1 , International Association of Science Hydrology Publications, (88), 597-604.

Chilingarian, G. V., E. C. Donaldson, and T. F. Yen, 1994, Subsidence due to fluid withdrawal: Elsevier Science.

Cohen, J. K., and R. M. Lewis, 1967, A ray method for the asymptotic solution of the diffusion equation: Journal of the Institute of Mathematics and its Applications., 3, 266-290.

Colazas, X. C, 1971, Subsidence, compaction of sediments and effects of water injection, Wilmington and Long Beach offshore fields: Masters of Science Thesis, University of Southern California.

Courant, R., and D. Hilbert, 1962, Methods of Mathematical Physics: Interscience.

Du, J., S. J. Brissenden, P. McGillivray, S. Bourne, P. Hofstra, E. J. Davis, W. H. Roadarmel, S. L. Wolhart, S. Marsic, R. Gusek, and C. A. Wright, 2005, Mapping reservoir volume changes during cyclic steam stimulation using tiltmeter based surface deformation measurements: 2005 SPE International Thermal Operations and Heavy Oil Symposium, CHOA 97848, Calgary, Alberta, Canada, 1-3 November 2005.

Dussealt, M. B., R. A. Bilak, and L. Rothenburg, 1993, Inversion of surface displacements to monitor in-situ processes: International Journal of Rock Mechanics and Mining Science, 30 , 1219-1222.
Evans, K. F., G. R. Holzhausen, and M. D. Wood, 1982, The geometry of a large-scale nitrogen gas hydraulic fracture formed in Devonian shale: An example of fracture mapping using tiltmeters: Society of Petroleum Engineers Journal, 22, 755-763.

Fielding, E. J., R. G. Blom, and R. M. Goldstein, 1998, Rapid subsidence over oil fields measured by SAR interferometry: Geophysical Research Letters, 25, 32153218 .

Ferretti, A., C. Prati, and F. Rocca, 2000, Nonlinear subsidence rate estimation using permanent scatterers in differential SAR interferometry: IEEE Transactions on Geoscience and Remote Sensing, 38, 2202-2212.

Ferretti, A., C. Prati, and F. Rocca, 2001, Permanent scatterers in SAR interferometry: IEEE Transactions on Geoscience and Remote Sensing, 39 , 8-20.

Friedlander, F. G. and J. B. Keller, 1955, Asymptotic expansions of solutions of $\left(\nabla^{2}+k^{2}\right) u=0$ : Communications of Pure and Applied Mathematics, 8, 387.

Geertsma, J., 1973, Land subsidence above compacting oil and gas reservoirs: Journal of Petroleum Technology, 25, 734-744.

Guilbot, J. and B. Smith, 2002, 4-D constrained depth conversion for reservoir compaction estimation: Application to Ekofisk field: The Leading Edge, 21, 302-308.

Hall, S. A., 2006, A methodology for 7D warping and deformation monitoring using time-lapse seismic data: Geophysics, 71, O21-O31.

Hatchell, P. and S. Bourne, 2005, Rocks under strain: Strain-induced time-lapse time shifts are observed for depleting reservoirs: The Leading Edge, 17, 1222-1225.

Hodgson, N., C. MacBeth, L. Duranti, J. Rickett, and K. Nihei, 2007, Inverting for reservoir pressure change using time-lapse time strain: Application to the Genesis Field, Gulf of Mexico, The Leading Edge, 26 , 649-652.

Hoversten, G. M., R. Gritto, J. Washbourne, and T. Daley, 2003, Pressure and fluid saturation prediction in a multicomponent reservoir, using combined seismic and electromagnetic imaging: Geophysics, 68, 1580-1591.

Kline, M., and I. W. Kay, 1979. Electromagnetic Theory and Geometrical Optics: Robert E. Krieger Publishing.

Kravtsov, Y. A., and Y. I. Orlov, 1990, Geometrical Optics of Inhomogeneous Media: Springer-Verlag.

Kumar, A., R. C. Ozah, M. Noh, G. Pope, K. Sepehrnoori, S. Bryant, and L. W. Lake, 2005, Reservoir simulation of $\mathrm{CO}_{2}$ storage in deep saline aquifers: Society of Petroleum Engineers Journal, 10, 336-343. 
Landro, M., 2001, Discrimination between pressure and fluid saturation changes from time-lapse seismic data: Geophysics, 66 , 836-844.

Landro, M., and J. Stammeijer, 2004, Quantitative estimation of compaction and velocity changes using $4 \mathrm{D}$ impedance and traveltime changes: Geophysics, 69, 949-957.

Lawson, C. L., and R. Hanson, 1974, Solving Least Squares Problems: Prentice-Hall.

Massonnet, D. and K. L. Feigl, 1998, Radar interferometry and its application to changes in the Earth's surface: Reviews of Geophysics, 36, 441-500.

Massonnet, D., T. Holzer, and H. Vadon, 1997, Land subsidence caused by East Mesa geothermal field, California, observed using SAR interferometry: Geophysical Research Letters, 24, 901-904.

Mossop, A., and P. Segall, 1999, Volume strain within the Geysers geothermal field: Journal of Geophysical Research, 104, 29113-29131.

Paige, C. C., and M. A. Saunders, 1982, LSQR: An algorithm for sparse linear equations and sparse linear systems: ACM Transactions of Mathematical Software, 8, 195-209.

Palmer, I. D., 1990, Uplifts and tilts at Earth's surface induced by pressure transients from hydraulic fractures: Society of Petroleum Engineers Production Engineering, 5, 324-332.

Parker, R. L., 1994, Geophysical inverse theory: Princeton University Press.

Peaceman, D. W., 1977, Fundamentals of Numerical Reservoir Simulation: Elsevier Scientific Publishing.

Rice, J. R., and M. P. Cleary, 1976, Some basic stress diffusion solutions for fluid-saturated elastic porous media with compressible constituents: Reviews of Geophysics and Space Physics, 14, 227-241.

Rickett, J., L. Duranti, T. Hudson, B. Regel, and N. Hodgson, 2007, 4-D time strain and the seismic signature of geomechanical compaction at Genesis: The Leading Edge, 26 , 644-647.

Roste, T., A. Stovas, and M. Landro, 2006, Estimation of layer thickness and velocity changes using $4 \mathrm{D}$ prestack seismic data: Geophysics, 71, S219-S234.

Sasagawa, G. S., W. Crawford, O. Eiken, S. Nooner, T. Stenvold, and M. A. Zumberge, 2003, A new sea-floor gravimeter: Geophysics, 68, 544-553.

Schmidt, D. A., and R. Burgmann, 2003, Time-dependent land uplift and subsidence in the Santa Clara valley, California, from a large interferometric synthetic aperture radar data set: Journal of Geophysical Research, 108, doi:10.1029/20002JB002267,4-1/4-13.
Segall, P., 1985, Stress and subsidence resulting from subsurface fluid withdrawal in the epicentral region of the 1983 Coalinga earthquake: Journal of Geophysical Research, 90, 6801-6816.

Shapiro, S. A., E. Rothert, V. Rath, and J. Rindschwentner, 2002, Characterization of fluid transport properties of reservoirs using induced microseismicity: Geophysics, 67, 212-220.

Spiess, F. N., C. D. Chadwell, J. A. Hildebrand, L. E. Young, G. H. Purcell, and H. Dragert, 1998, Precise GPS/Acoustic positioning of seafloor reference points for tectonic studies: Earth and Planetary Science Letters., 108, 101-112.

Stancliffe, R. P. W., and M. W. A. van der Kooij, 2001, The use of satellite-based radar interferometry to monitor production activity at the Cold Lake heavy oil field, Alberta, Canada: American Association of Petroleum Geologists Bulletin, 85, 781-793.

Staples, R., J. Ita, R. Burrell, and R. Nash, 2007, Monitoring pressure depletion and improving geomechanical models of the Shearwater Field using 4D seismic: The Leading Edge, 26 , 636-642.

Tura, A., T. Barker, P. Cattermile, C. Collins, J. Davis, P. Hatchell, K. Koster., P. Schutjens, and P. Willis, 2005, Monitoring primary depletion reservoirs using amplitudes and time shifts from high-repeat seismic surveys: The Leading Edge, 24, 1214-1221.

Tura, A., and D. E. Lumley, 1999, Estimating pressure and saturation changes from time-lapse AVO data: 61 st Annual Conference of the European Association of Geoscientists and Engineers, Extended Abstracts, 1-38.

Vasco, D. W., 2004, Estimation of flow properties using surface deformation and head data: A trajectorybased approach: Water Resources Research, 40, doi:10.1029/2004WR003272, 1-14.

Vasco, D. W., L. R. Johnson, and N. Goldstein, 1988, Using surface deformation and strain observations to determine deformation at depth, with an application to Long Valley Caldera, California: Journal of Geophysical Research, 93 , 3232-3242.

Vasco, D. W., K. Karasaki, and L. Myer, 1998. Inversion of surface tilt caused by fluid migration: Journal of Geotechnical and Geoenvironmental Engineering, 124, 29-37.

Vasco, D. W., K. Karasaki, and C. Doughty, 2000a, Using surface deformation to image reservoir dynamics: Geophysics, 65 , 132- 147.

Vasco, D. W., K. Karasaki, and H. Keers, 2000b, Estimation of reservoir properties using transient pressure data: An asymptotic approach: Water Resources Research, 36, 3447-3465. 
Vasco, D. W., K. Karasaki, and K. Kishida, 2001, A coupled inversion of pressure and surface displacement: Water Resources Research, 37, 3071-3089.

Vasco, D. W., A. Ferretti, and F. Novali, 2008, Estimating permeability from quasi-static deformation: Temporal variations and arrival time inversion: Geophysics, (in press).

Vasco, D. W., and A. Ferretti, 2005, On the use of quasistatic deformation to understand reservoir fluid flow: Geophysics, 70, O13-O27.

Virieux, J., C. Flores-Luna, and D. Gibert, 1994, Asymptotic theory for diffusive electromagnetic imaging: Geophysical Journal International, 119, 857-868.

Wang, R., and H.-J., Kuempel, 2003, Poroelasticity: Efficient modeling of strongly coupled, slow deformation processes in a multilayered half-space: Geophysics, 68, 705-717.

Wright, C. A., 1998, Tiltmeter fracture mapping: From the surface and now downhole: Petro. Eng. Int., January, 50-63.

Wright, C. A., E. J. Davis, W. A. Minner, J. F. Ward, L. Weijers, E. J. Schell, and S. P. Hunter, 1998, Surface tiltmeter fracture mapping reaches new depths 10,000 feet and beyond?: Society of Petroleum Engineers, 39919, April.

\section{FIGURE CAPTIONS}

Figure 1. Range velocity estimates derived from Interferometric Synthetic Aperture Radar (InSAR) data. Positive values indicate motion towards the satellite. The upper panel displays values associated with Track 294 while the lower panel displays values associated with Track 65 .

Figure 2. Range velocities obtained by averaging observations from Tracks 65 and 294. The trajectory of the injection well KB-502 is indicated by the solid line in the figure. Positive values indicate points moving towards the reference point in space.

Figure 3. Range changes for four time intervals. The trajectory of the injection well KB-502 is indicated by the solid line in the figure.

Figure 4. Pressure estimates for a model in which all of the changes are confined to the $20 \mathrm{~m}$ thick reservoir interval.

Figure 5. Pressure estimates for a model in which changes are allowed up to $190 \mathrm{~m}$ above the layer. The four panels correspond to time intervals displayed in Figure 3.

Figure 6. Contour plot of the phase estimates obtained from the time at which the peak rate of pressure change occurred [see equation (19)].

Figure 7. Sampled field of phase estimates, where the size of the unfilled squares indicates the phase value. The largest square corresponds to a value of $20 \sqrt{\text { days. The }}$ trajectories $\mathbf{X}$, plotted as solid lines in this figure, are computed by marching down gradient from the phase field.

Figure 8. Logarithm of the permeability multiplier. Large, positive values indicate higher permeabilities.

Figure 9. Observed arrival time residual plotted against calculated arrival time residual. The residual is the remainder after the reference arrival time is subtracted. For a perfect fit the points would lie on the $45^{\circ}$ line.

Figure 10. Diagonal elements of the model parameter resolution matrix. For values near 1 the permeability estimate may be resolved in isolation from the surrounding estimates. For values near 0, there is considerable tradeoff with other permeability estimates. 

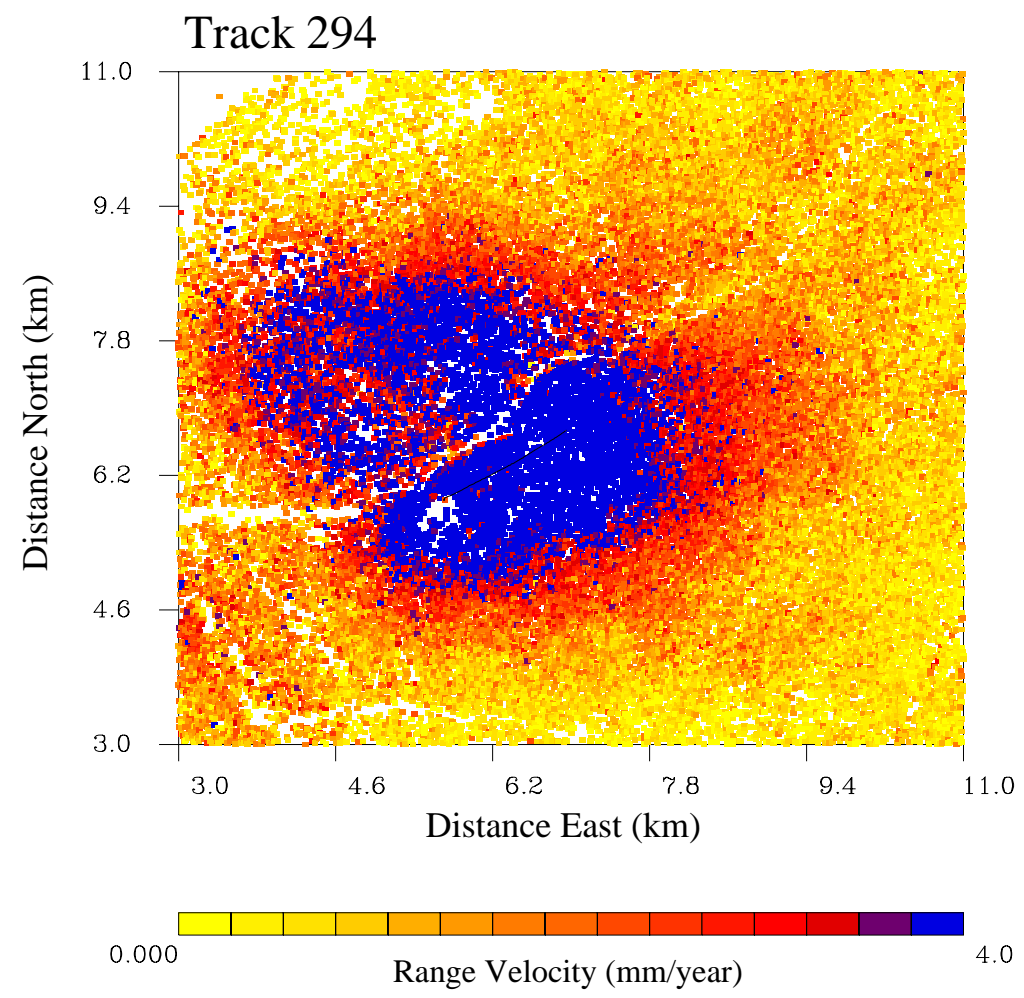

\section{Track 65}
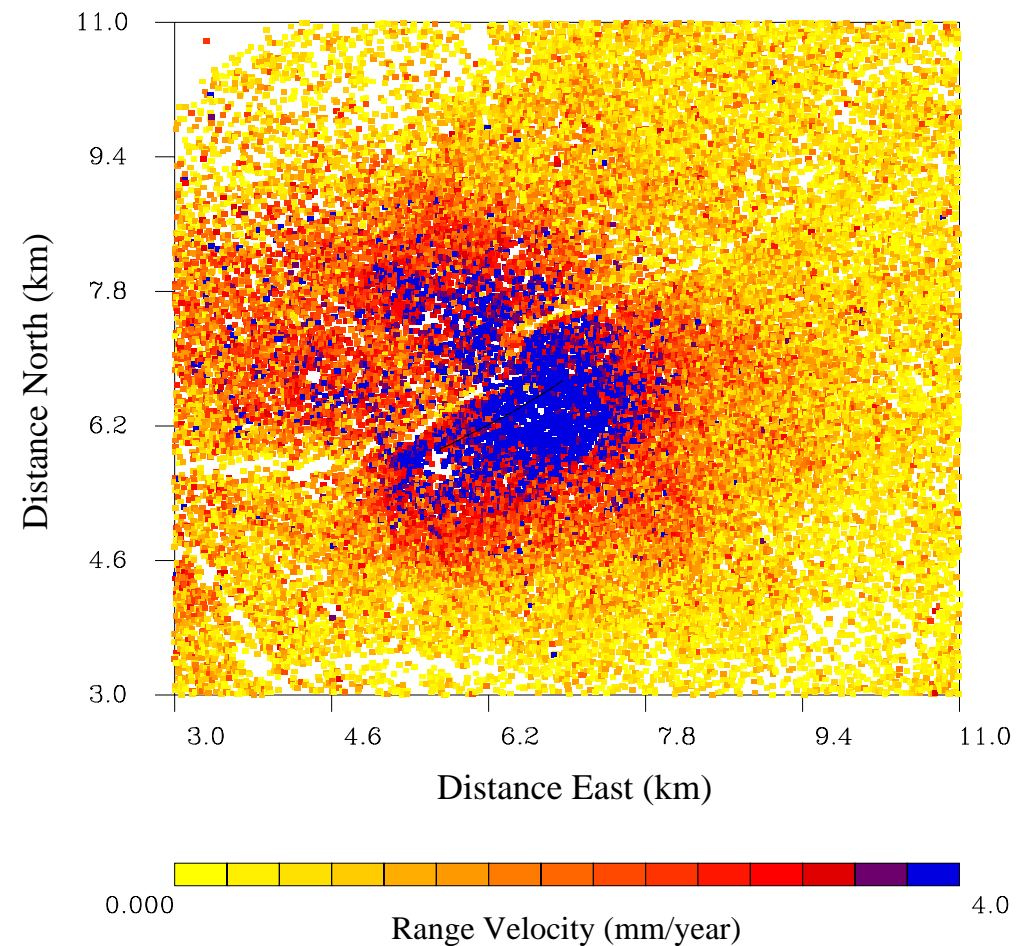

Figure 1. 


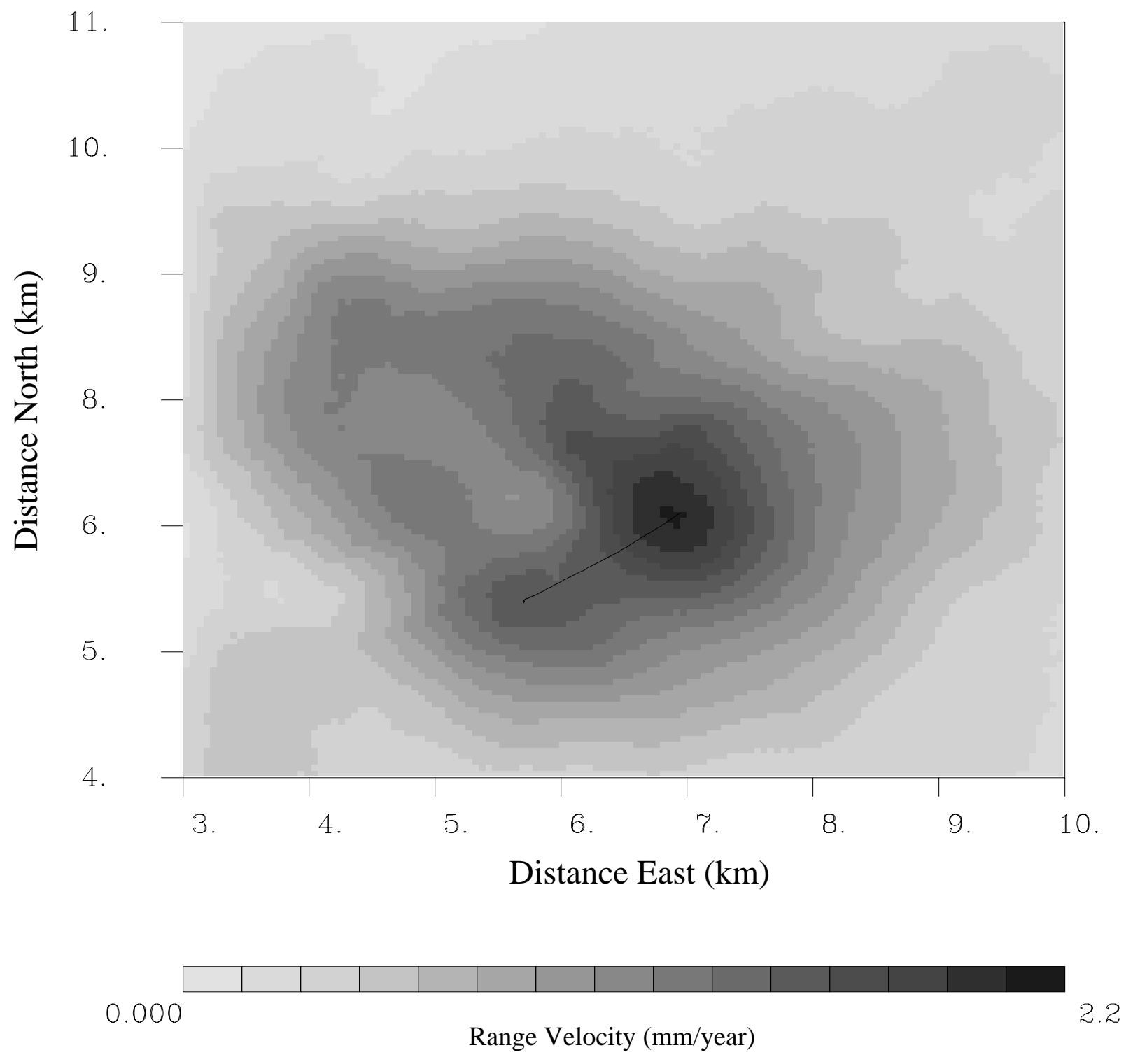

Figure 2 . 

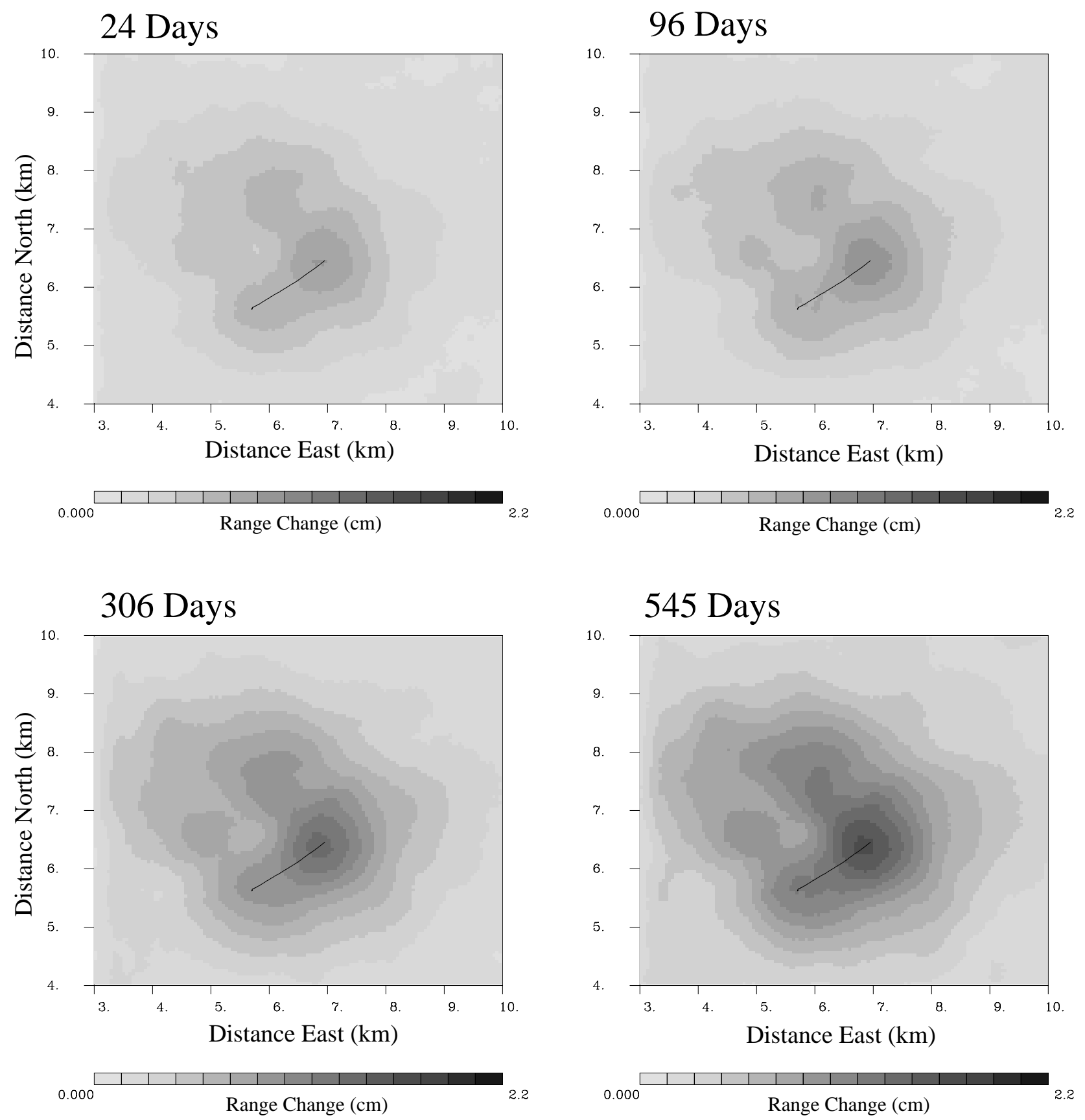


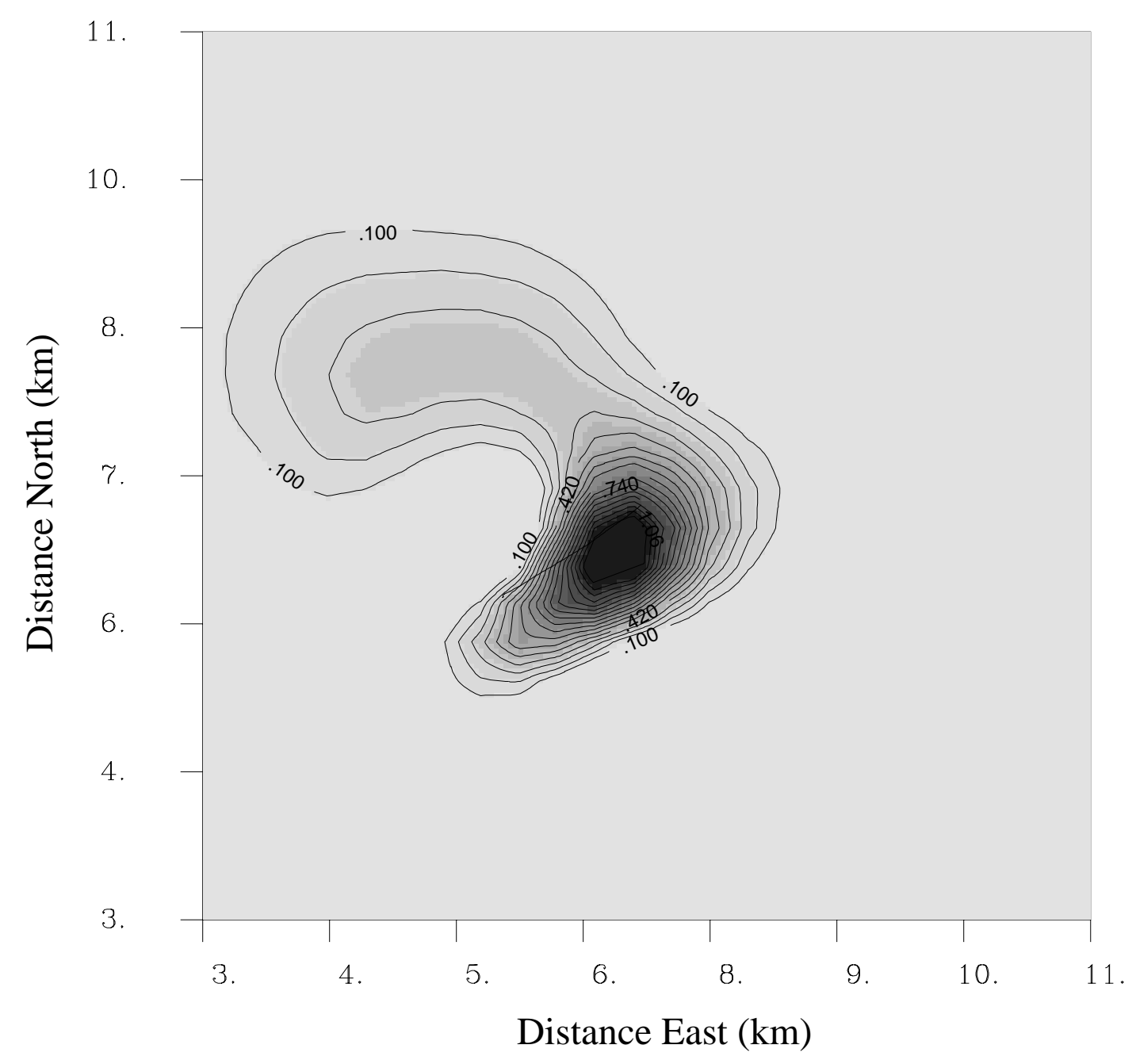

Figure 4. 

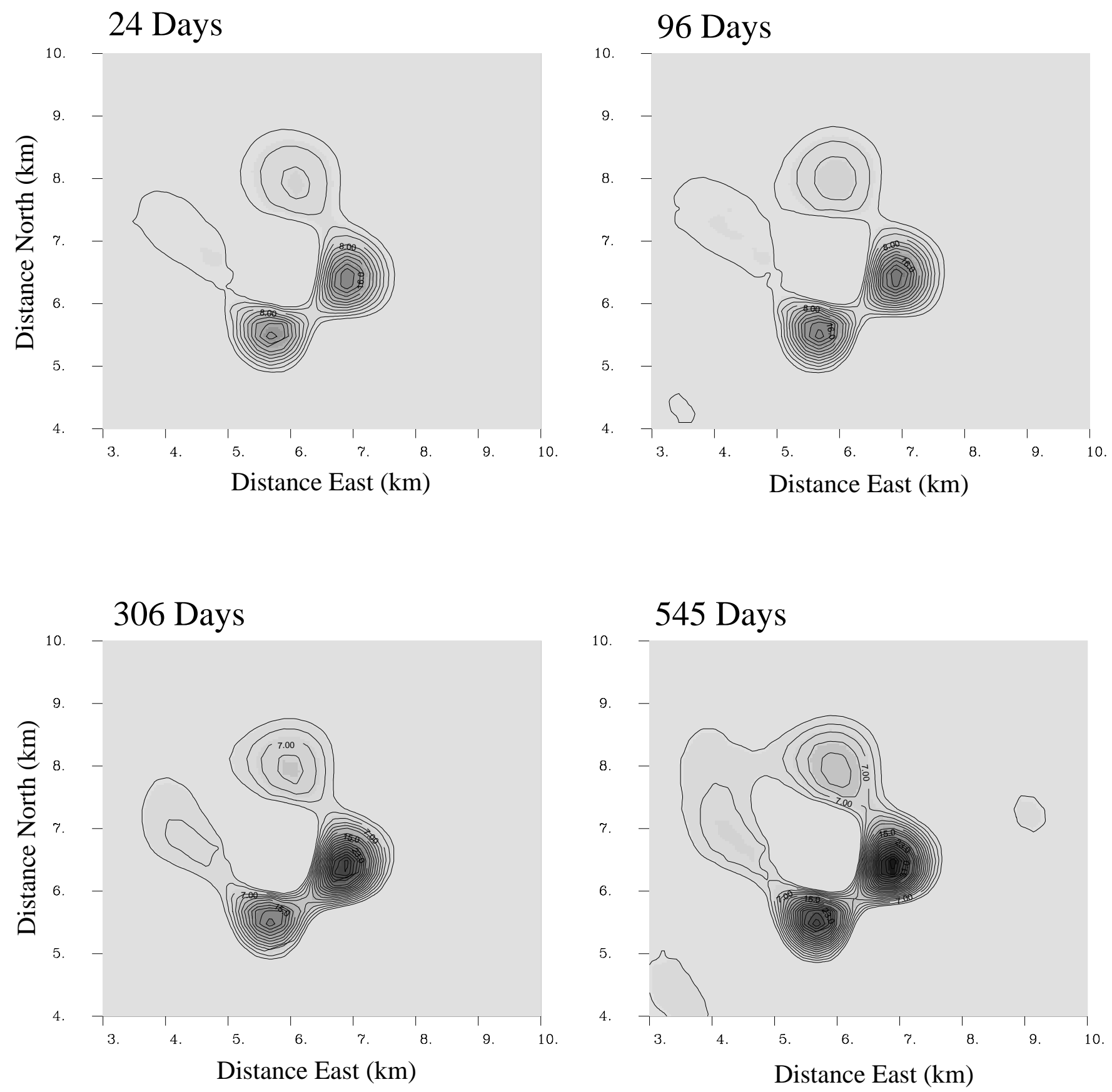

Figure 5. 


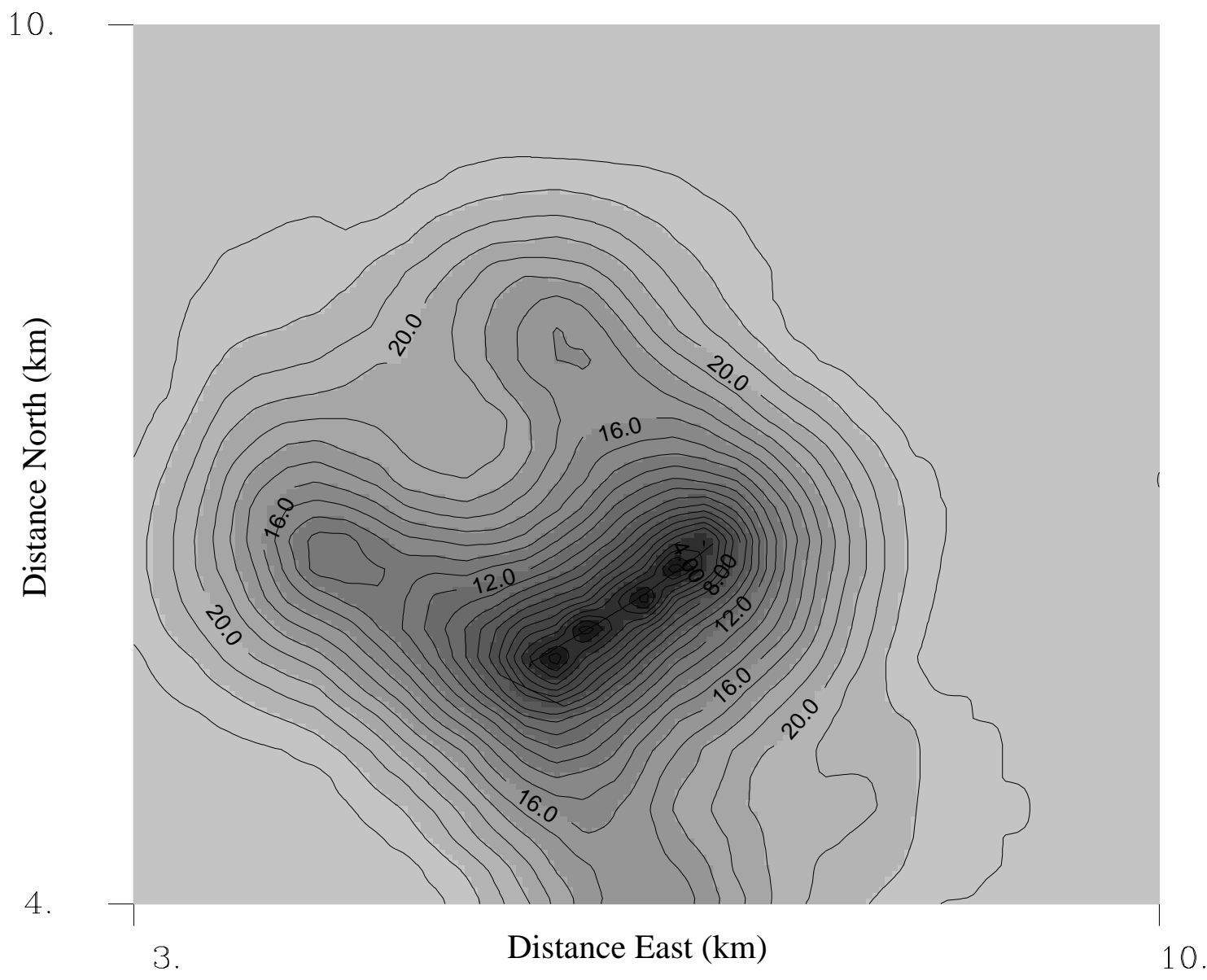

0 .

Square Root of Arrival Time (days)

30.

Figure 6. 


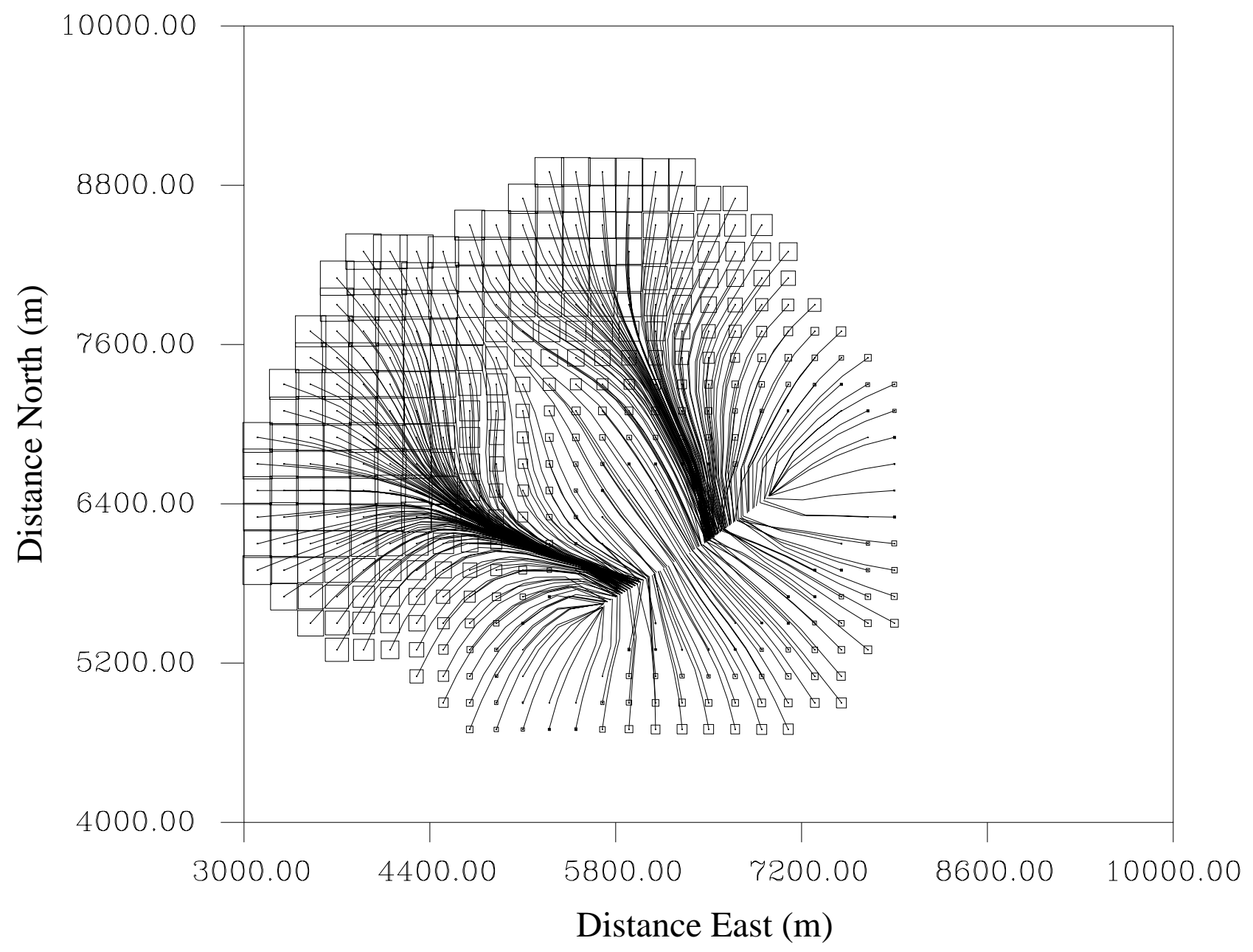

Figure 7 . 


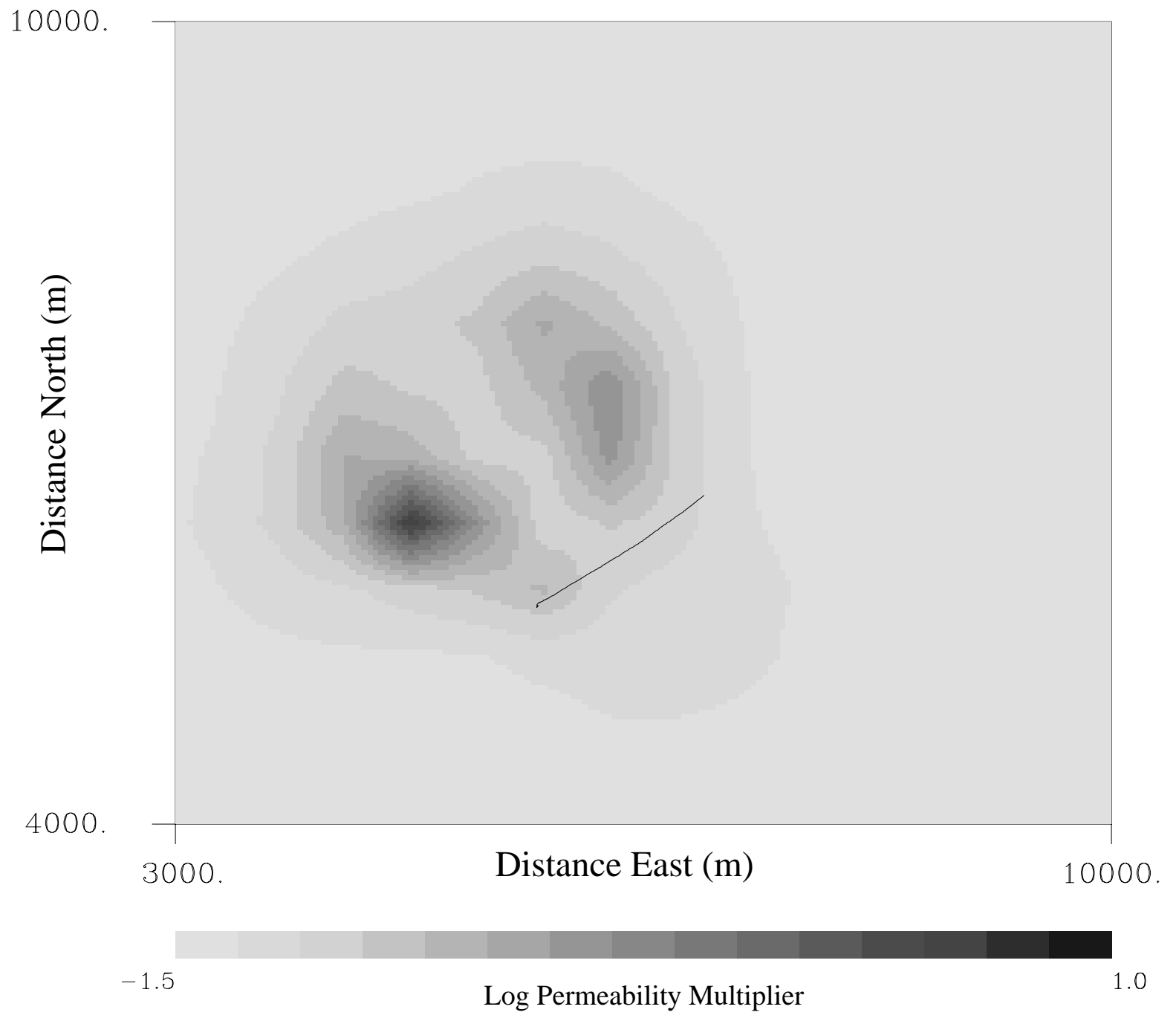

Figure 8 . 


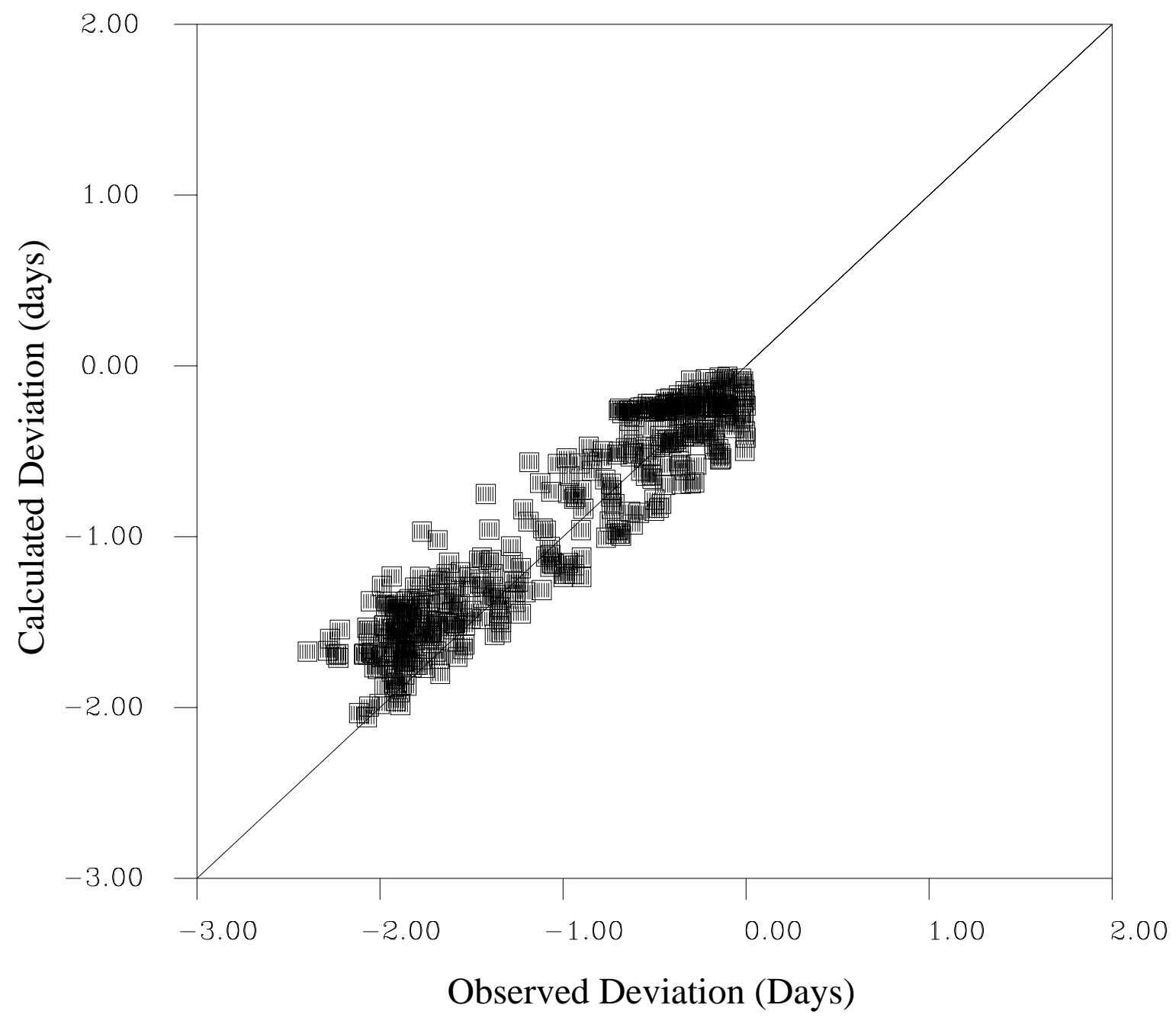

Figure 9. 


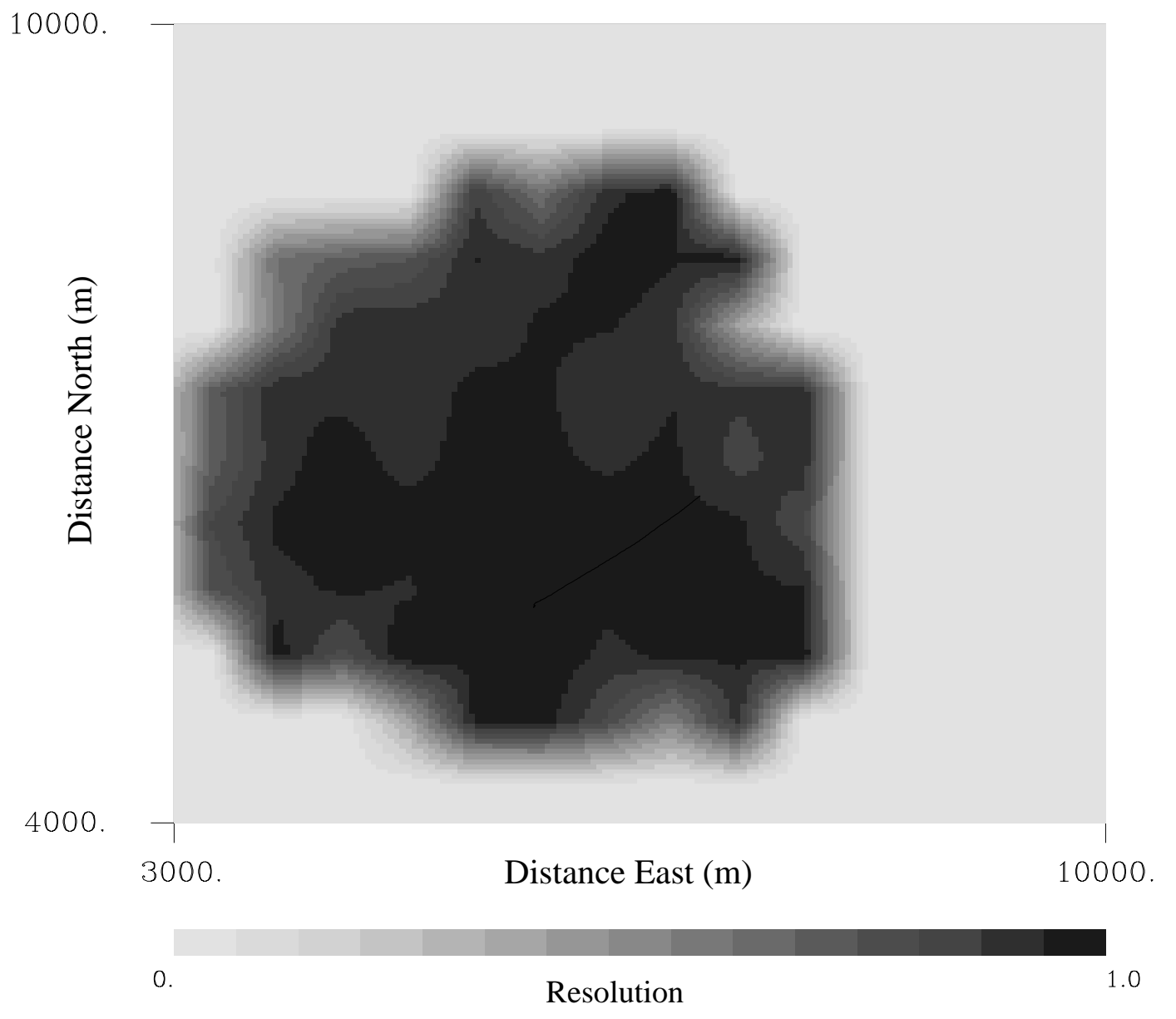

Figure 10. 